\title{
Fission diamond detector tests at the ISIS spallation neutron source
}

\author{
M. Rebai ${ }^{\mathrm{a} *}$, C. Andreani ${ }^{\mathrm{b}}$, A. Fazzi ${ }^{\mathrm{c}}$, C.D. Frost ${ }^{\mathrm{d}}$, L. Giacomelli $^{\mathrm{a}}$, G. Gorini ${ }^{\mathrm{a}}$, E. Milani ${ }^{\mathrm{e}}$, E. Perelli \\ $\mathrm{Cippo}^{\mathrm{a}}$, A. Pietropaolo ${ }^{\mathrm{b}}$, G. Prestopino ${ }^{\mathrm{e}}$, E. Schooneveld ${ }^{\mathrm{d}}$, M. Tardocchi ${ }^{\mathrm{a}}$, C. Verona $^{\mathrm{e}}$ and G. Verona \\ Rinati $^{\mathrm{e}}$
}

${ }^{a}$ CNISM, Dipartimento di Fisica, Università degli Studi di Milano-Bicocca, and Istituto di Fisica del Plasma, Associazione EURATOM-ENEA-CNR, Milano, Italy

${ }^{\mathrm{b}}$ Dipartimento di Fisica, Centro NAST and CNISM, Università degli Studi di Roma Tor Vergata, Via della Ricerca Scientifica 1, 00133 Roma, Italy

${ }^{\mathrm{c}}$ Energy Department, Politecnico di Milano, Via Lambruschini 4, 20156 Milano, Italy

${ }^{\mathrm{d} S T F C}$, ISIS facility, Rutherford Appleton Laboratory, Chilton Didcot Oxfordshire, OX11 0QX, United Kingdom

e Dipartimento di Ingegneria Meccanica, Università degli Studi di Roma Tor Vergata, Via del Politecnico 1, 00133 Roma, Italy

A compact device for monitoring of fast neutron fluxes is presented. The device is based on single crystal diamond obtained by the Chemical Vapor Deposition technique coupled to a uranium converter foil where neutron interaction results in emission of charged particles detected inside the diamond. Thermal and fast neutrons are detected using natural uranium containing both ${ }^{235} \mathrm{U}$ and ${ }^{238} \mathrm{U}$. Biparametric (pulse height and time of flight) data collection was used at the ISIS pulsed neutron source to distinguish events from ${ }^{235} \mathrm{U},{ }^{238} \mathrm{U}$ and from carbon break-up reactions inside the diamond.

The interaction of high energy cosmic rays with the earth's atmosphere generates a flux of secondary particles including high energy neutrons [1]. Single Event Upsets (SEU) can occur when a high energy neutron, interacting in the sensitive region of an electronic device, disrupts its correct operation [2]. Spallation neutron sources can provide a neutron energy spectrum similar to the atmosferic one, with much higher fluxes for accelerated neutron testing. A new beamline named CHIPIR for SEU studies will be constructed at the ISIS spallation neutron source [3] where experience on neutron irradiation testing is growing $[4,5]$ and new concepts for fast neutron beam monitors are being tested [6]. One line of devel-

\footnotetext{
*This work was supported within the CNR-CCLRC Agreement No.01/9001 concerning collaboration in scientific research at the spallation neutron source ISIS. The financial support of the Consiglio Nazionale delle Ricerche in this research is hereby acknowledged.
}

opment is the monitoring of the neutron flux on the electronic board or chip which requires compact detectors. Diamond detectors are suitable candidates [7]. They are normally used as Singlecrystal Diamond Detectors [8] in which case neutron detection takes place via carbon break-up reactions in the diamond itself. In this paper a composite detector called Fission Diamond Detector (FDD) is presented where a thin $(25 \mu \mathrm{m}$ thick and $4 \mathrm{~mm}^{2}$ ) natural uranium foil is placed in front of a diamond detector grown by microwave-assisted chemical vapour deposition $[9,10]$. Heavy fragments from neutron induced fission in uranium deposit a large amount of energy (tens of $\mathrm{MeV}$ ) in the diamond. Fission events are thus recorded together with intrinsic events due to ${ }^{12} \mathrm{C}(\mathrm{n}, \alpha){ }^{9} \mathrm{Be}$ and ${ }^{12} \mathrm{C}(\mathrm{n}, 3 \alpha) \mathrm{n}$ and ${ }^{12} \mathrm{C}(\mathrm{n}, \mathrm{n}){ }^{12} \mathrm{C}$ reactions in the diamond. Fission events due to fast neutrons can be separated from other detection events, making 
the FDD a candidate fast neutron flux monitor in CHIPIR.

The neutron production at ISIS relies upon spallation reactions induced by $800-\mathrm{MeV}$ proton bunches accelerated through a synchrotron [11]. Protons are extracted into two bunches (about 320 ns apart) and delivered to a TungstenTantalum target. The estraction frequency is either 10 or $50 \mathrm{~Hz}$ and the time-average current is about $200 \mu \mathrm{A} / \mathrm{h}$. The ROTAX beamline used in the present tests, has a methane moderator at $95 \mathrm{~K}$ providing a fast neutron spectrum scaling roughly as $1 / E_{\mathrm{n}}$. The FDD was placed at a distance of $15.5 \mathrm{~m}$ from the moderator and the diamond bias voltage was $30 \mathrm{~V}$. The natural uranium foil was placed in front of the aluminum contact at a distance of $2 \mathrm{~mm}$ leaving a small air gap to ensure insulation without preventing fission fragments from reaching the diamond volume.

The electronic signal chain consisted of a custom made charge preamplifier (integration time of 500 ns), a Timing Filter Amplifier and a N1728 CAEN Waveform Digitizer [12]. Data were collected in biparametric mode, where pulse height and time of each detection event are stored. The CAEN firmware implements a trapezoidal filter [13] to determine the pulse height. Neutron time of flight is determined off line with reference to a proton pulse logic signal which is also stored at every ISIS pulse.

Data were collected for 24 hours, for an integrated proton current of $3578 \mu \mathrm{A} / \mathrm{h}$.

The data recorded by the FDD detector are shown in figure 1 as a scatter plot. On the vertical axis the pulse height is shown in terms of $E_{\mathrm{d}}$, the equivalent energy deposited in the detector. On the horizontal axis the time of flight, $t_{\mathrm{ToF}}$, is plotted.

The recorded events can be divided in three areas as shown in the diagram.

i. Flight times in excess of $1500 \mathrm{~ns}$ are associated with neutrons well below the $\mathrm{U}^{238}$ fission threshold. These events are due to ${ }^{235} \mathrm{U}$ fission or to $\alpha$-particles from uranium decay.

ii. Events with $E_{\mathrm{d}}>20 \mathrm{MeV}$ and $t_{\mathrm{ToF}}<1500 \mathrm{~ns}$ must be associated with heavy fission frag-

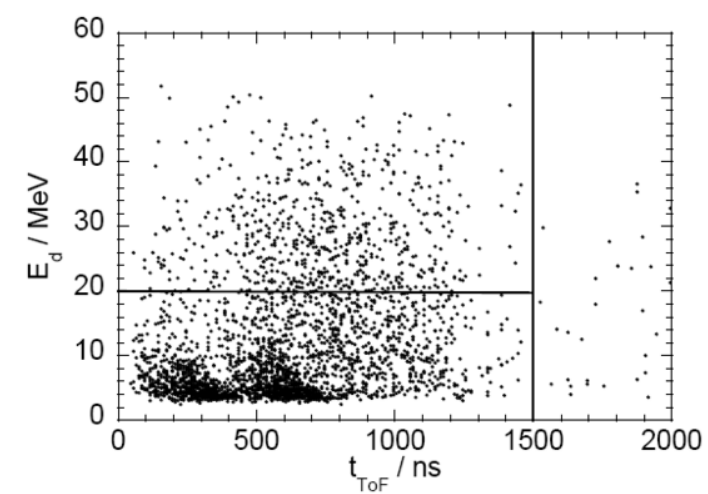

Figure 1. Biparametric (pulse height $E_{\mathrm{d}}$ and time of flight $t_{\mathrm{ToF}}$ ) scatter plot of data recorded with the FDD detector in the ROTAX neutron beam line at ISIS.

ments from the uranium foil since deposited energies $E_{\mathrm{d}}>20 \mathrm{MeV}$ are not observed when the uranium foil is removed from the FDD [8]. The contribution of ${ }^{235} \mathrm{U}$ fission is comparatively low and can be estimated from the event rate measured above $1500 \mathrm{~ns}$; it is thus reasonable to assume that ${ }^{238} \mathrm{U}$ fission by fast neutrons is responsible for the events with $E_{\mathrm{d}}>20 \mathrm{MeV}$ and $t_{\mathrm{ToF}}<1500 \mathrm{~ns}$.

iii. At deposited energies $E_{\mathrm{d}}<20 \mathrm{MeV}$ and flight times $t_{\mathrm{ToF}}<800 \mathrm{~ns}$ the dominant reaction mechanism is carbon break-up in diamond, see [8].

The ToF spectrum derived from the data of figure 1 with $E_{\mathrm{d}}>20 \mathrm{MeV}$ is shown in figure 2 . The TOF spectral shape is significantly different from the one obtained integrating events of $E_{\mathrm{d}}<20 \mathrm{MeV}$ [8]. This is due to the dominant contribution of neutrons in the energy range $1 \mathrm{MeV}-5$ $\mathrm{MeV}$ which are detected through the induced fission reactions in ${ }^{238} \mathrm{U}$ since they are below the threshold for reactions with ${ }^{12} \mathrm{C}$. In order to understand the spectrum in more detail a model calculation can be made. For a pulsed neutron source where all neutrons leave the moderator simultaneously the number of recorded events per unit $t_{\mathrm{ToF}}$ can be written as:

$N\left(t_{\mathrm{ToF}}\right)=K \cdot \sigma(E) \cdot \phi(E) \cdot \frac{d E}{d t_{\mathrm{ToF}}}$

where $K$ is a proportionality constant, $\sigma$ is the ${ }^{238} \mathrm{U}$ fission cross section, $\phi$ is the neutron flux 


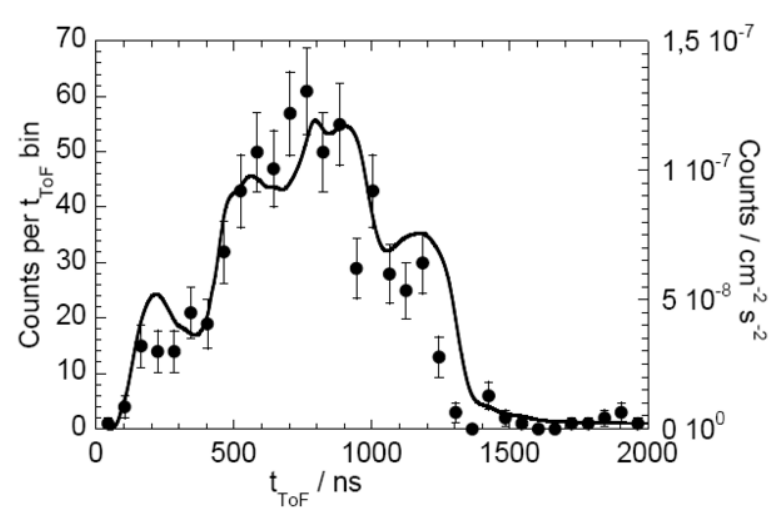

Figure 2. ToF spectrum derived from the data of figure 1 with $E_{\mathrm{d}}>20 \mathrm{MeV}$. Also shown is a simulated $t_{\text {ToF }}$ spectrum (see text). The error bars are statistical.

per unit neutron energy (e.g. in units of $\mathrm{cm}^{-2}$ $\mathrm{s}^{-1} \mathrm{MeV}^{-1}$ ) on the uranium foil and $d E / d t_{\mathrm{ToF}}$ is the Jacobian of the energy- $t_{\text {ToF }}$ transformation.

In our case the time structure of the fast neutrons leaving the moderator can be described by the measured proton pulse structure, i.e. two Gaussian bunches 320 ns apart. The line in figure 2 is the result of the convolution of the proton pulse structure with the result of Eq. 1 where a reference MCNPX [14] neutron spectrum for the ROTAX beam line was used. The fission cross section from [15] provides values up to $E_{\mathrm{n}}=200$ $\mathrm{MeV}$. The agreement between data and simulation after adjustment of the constant $K$ is rather good and confirms the identification of the selected events with ${ }^{238} \mathrm{U}$ fission events with little or no contribution from other processes or other types of radiation. It therefore seems feasible to proceed with the development of an FDD optimized for the CHIPIR application. The absolute efficiency of the FDD is much lower than ordinary fission chambers since it scales with the detector surface. It is also substantially lower than Thin Film Breakdown Counters [16] for the same reason. Otherwise the FDD response should be similar to other uranium-based detectors.

In conclusion, first tests of a Fission Diamond Detector prototype at the ISIS pulsed neutron source show that the FDD concept has the potential to be used as a compact fast neutron monitor in irradiation experiments. Biparametric (pulse height and time of flight) data collection is essential for a correct separation of events from ${ }^{235} \mathrm{U}$, ${ }^{238} \mathrm{U}$ and from carbon break-up reactions inside the diamond. The FDD is also sensitive to thermal neutrons, a feature that could also find application in accelerated neutron testing of electronic devices.

\section{REFERENCES}

1. J.F. Ziegler et al., IBM J. Res. Devel. 40 no. 1 3-1, Jan. 1996.

2. P.E. Dodd and L.W. Massengill, IEEE Trans. Nucl. Sci. 50 no. 3 583-602 Jun. 2003.

3. http://www.isis.stfc.ac.uk/instruments/ChipIr.

4. C. Andreani et al., Appl. Phys. Lett. 92 114101 (2008).

5. M. Violante et al., IEEE Trans. Nucl. Sci. 54 1184 (2007).

6. R. Bedogni et al., Nucl. Inst. and Meth. A 612 (2009) 143-148.

7. D. Lattanzi et al., Fus. Eng. and Des. 84 Issues 7-11 (2009) 1156-1159.

8. L. Giacomelli et al., Nucl. Phys. B Proceeding of IPRD10 (2010).

9. M. Marinelli et al., Appl. Phys. Lett. 753216 (1999).

10. M. Marinelli et al., Journ. Appl. Phys. 89 1430 (2001).

11. http://www.isis.stfc.ac.uk/.

12. CAEN Nuclear Physics, Technical information manual - MOD. N1728A/B $4 \mathrm{CH}$ $100 \mathrm{MHz}$ flash ADC - Manual Revision no. 5, NPO: 00118/04:N1728x.MUTx/05 (2007).

13. V.T. Jordanov, G.F. Knoll, Nucl. Instr. and Meth. A 345 (1994) 337-345.

14. https://mcnpx.lanl.gov/.

15. http://www-nds.iaea.org/exfor/exfor.htm.

16. A.N. Smirnov et al., Report RAL-TR2010-024, Rutherford Appleton Laboratory, Chilton (UK) 2010. 